Gravitational waves from eccentric intermediate mass binary black hole mergers

This article has been downloaded from IOPscience. Please scroll down to see the full text article.

2009 Class. Quantum Grav. 26204008

(http://iopscience.iop.org/0264-9381/26/20/204008)

View the table of contents for this issue, or go to the journal homepage for more

Download details:

IP Address: 194.94.224.254

The article was downloaded on 14/06/2010 at 10:07

Please note that terms and conditions apply. 


\title{
Gravitational waves from eccentric intermediate mass binary black hole mergers
}

\author{
Birjoo Vaishnav ${ }^{1}$, Ian Hinder ${ }^{2}$, Deirdre Shoemaker ${ }^{3}$ and \\ Frank Herrmann ${ }^{4}$ \\ ${ }^{1}$ Center for Gravitational Wave Astronomy, University of Texas at Brownsville, TX 78520, USA \\ 2 Max-Planck-Institut fuer Gravitationsphysik, Albert-Einstein-Institut, Potsdam-Golm, Germany \\ ${ }^{3}$ Center for Relativistic Astrophysics, School of Physics, Georgia Institute of Technology, \\ Atlanta, GA 30332, USA \\ ${ }^{4}$ Department of Physics, University of Maryland, College Park, MD 20742, USA
}

Received 1 May 2009, in final form 7 September 2009

Published 6 October 2009

Online at stacks.iop.org/CQG/26/204008

\begin{abstract}
Owing to the difficulty of direct observation, mergers of intermediate-mass black hole binaries are relatively less understood compared to stellar-mass binaries; however, the gravitational waves from their last few orbits and ringdown fall in the band of ground-based detectors. Because the typical source is expected to circularize prior to entering LIGO or VIRGO's range, inspiral searches concentrate on circularized binaries. It is possible that events will be missed if there are sources with residual eccentricity. We study the variation of the signal to noise present in the dominant mode of the eccentric evolutions as a function of mass and eccentricity and also the relative contribution of the signal in the various spherical harmonic modes. The energy radiated in gravitational waves increases with eccentricity until the eccentricity becomes too high, leading to plunging trajectories, at which point the energy radiated decreases. This enhancement of the energy for initial eccentricities near the transition value translates into larger signal-to-noise ratios. Consequently despite the anticipated loss in the signal-to-noise ratio due to the use of quasicircular detection templates, some eccentric signals potentially may be seen farther out than others.
\end{abstract}

PACS numbers: 04.25.dg, 04.30.Db

(Some figures in this article are in colour only in the electronic version)

\section{Introduction}

With ground-based gravitational-wave detectors at their initial design sensitivity and advancing toward sensitivities that may enable multiple detections of gravitational sources, it is important 
to examine the reach of present detection methods. The templates for non-spinning binaries used in inspiral searches of LIGO-VIRGO data use high-order post-Newtonian waveforms and analytic models; however, the breakthroughs in numerical relativity for binary black hole (BBH) mergers offer new opportunities to incorporate the merger and ringdown parts coherently in the search for BBH signals.

Among the sources for the ground-based detectors, the mergers of intermediate mass black holes (IMBHs) can carry the highest signal to noise ratio (SNR) since they would merge at LIGO's most sensitive frequencies. While there are at least a few known binary systems of stellar mass black holes from which estimates of binary formation rates can be made, the claims for IMBH, with masses in the range of $10^{2} M_{\odot} \leqslant M \leqslant 10^{4} M_{\odot}$, are not as well established. There are, however, various merger mechanisms proposed for IMBH mergers $[1,2]$. A gravitational wave detection of such systems would be the smoking gun for the existence of IMBHs and for the formation mechanism of the binaries. For example, some of the proposed scenarios for forming intermediate mass black hole binaries (IMBBH) indicate the possibility of high residual eccentricities in the binary orbit, even close to the merger [3], at least for LISA frequencies. The plausibility of finding significant eccentricities in LIGO band is still under investigation [4].

For stellar mass binaries, most astrophysical scenarios predict the eccentricity to be negligible and hence the inspiral searches assume the signal to be quasi-circular. Owing to this assumption of quasi-circularity, a possible signal from an eccentric binary may be lost by the searches. The extent of such losses was first investigated by Martel and Poisson, [5]. They found that for a given eccentricity the loss of the signal-to-noise ratio (SNR) decreased with increasing mass and for a given mass the loss of SNR increased with increasing eccentricity. Recently, this problem was revisited by Tessmer and Gopakumar [6] using higher order PN waveforms than the ones used earlier. They found that the efficiency of post-Newtonian quasi-circular waveforms in detecting the 2.5 PN eccentric waveforms presented in their paper varied for waveforms calculated by different prescriptions. Cokelaer and Pathak [7] revisit that work for current and future ground-based detectors and found that eccentricities less than 0.05 had fitting factors $\geqslant 0.95$ and could be captured by circular templates for binaries of total mass $[2,45] M_{\odot}$.

Our aim in the present paper is to take the first steps toward extending these previous analyses to IMBBHs with total masses $M / M_{\odot} \geqslant 30$. At this mass-range, the merger and ringdown of the binary dominates the SNR allowing us to use numerical relativity waveforms without matching to post-Newtonian ones. We investigate the SNR for waveforms of equalmass $\left(m_{1}=m_{2} \equiv M / 2\right)$, non-spinning, eccentric binaries during their last few orbits and merger. These waveforms were computed with the MayaKranc code [8, 9]. The work presented here is a preliminary study and a future report will include the fitting factors of these eccentric waveforms with noneccentric template waveforms to test the effectiveness of circular templates at detecting eccentric IMBBHs [10].

The notation and formulae are explained in section 2, our results are presented in section 3 and the conclusions in section 4 , along with caveats and future work.

\section{Notation and formalism}

\subsection{Merger waveforms from numerical relativity}

We outline the spectra and some details of the numerical waveforms, and then outline the frequencies over which these waveforms could be a reliable representation of a LIGO 

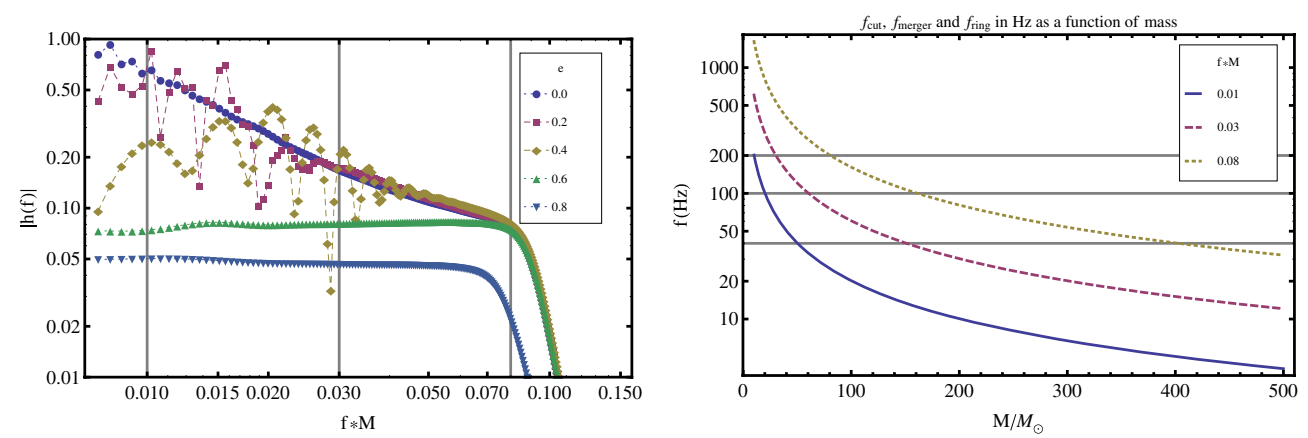

Figure 1. Left panel: the spectra for strain with $|h(f)|$ representing both $\sim\left|\tilde{h}_{+}(f)\right| \simeq \tilde{h}_{\times}(f)$ from various eccentricities to illustrate four distinct regions of the spectrum initial data/early inspiral, late inspiral, circularized plunge/merger and ringdown. Right panel: the frequency in $\mathrm{Hz}$ corresponding to the three regions is demarcated in the spectra to show the scaling with mass. The horizontal lines indicate the lower cutoff frequency for iLIGO $40 \mathrm{~Hz}$, and the sweet spot region between 100 and $200 \mathrm{~Hz}$.

signal. The waveforms are equal-mass, non-spinning binaries with initial eccentricities of $e=\{0.0,0.1,0.2,0.3,0.4,0.5,0.6,0.7,0.8\}$.

2.1.1. Strain waveforms in Fourier domain: Our numerical waveforms are calculated from a three-dimensional, general relativistic evolution in terms of the Newman-Penrose $\Psi_{4}$, where $r m \Psi_{r}(t, r)=\sum_{\ell m-2} C_{\ell m}(t, r)_{-2} Y_{\ell m}(\theta, \phi)$, and $r$ is the radius of the coordinate sphere on which $\Psi_{4}$ is computed. The relationship between $\Psi_{4}$ at future null infinity and the actual amplitude of the gravitational wave, $h(t)$, is given in terms of the two polarizations of the gravitational waves, $h_{+}(t)$ and $h_{\times}(t)$, as $\Psi_{4}(t)=\frac{\mathrm{d}^{2}}{\mathrm{~d} t^{2}}\left(h_{+}(t)-\mathrm{i} h_{\times}(t)\right)$, which will be approximately correct at a large extraction radius. The impact of extraction radius as well as the numerical errors on detection of eccentric mergers is addressed elsewhere [11].

To carry out the analysis, we compute the Fourier transform of the quantities $h_{+}(t)$ and $h_{\times}(t)$. These quantities are computed directly from the Fourier transform of the real and imaginary parts of $\Psi_{4}$ to yield $h_{+}(f)$ and $h_{\times}(f)$ respectively, for example, $\tilde{h}_{+}(f)=\mathcal{F}\left(\operatorname{Re}\left(\Psi_{4}\right)\right)(f) /\left(-4 \pi^{2} f^{2}\right)$. This avoids issues regarding integration constants that arise from the time domain integration of $\Psi_{4}$ [12].

In previous work, [8], we studied the set of eccentric waveforms used here for how the binary circularizes during the non-linear portion of the coalescence. Figure 1 shows the spectra of a selection of the eccentric runs. The three vertical lines of the figure to the left illustrate regions of the evolution.

The first and second regions indicate the modulations that are due to the different initial eccentricities each evolution possessed. The third region shows the 'circularization' of all the binaries prior to merger. The final region shows that the black holes ring down to the same final values of mass and spin. This demonstrates the results of [8] which reported that the binaries with $e \leqslant 0.3$ formed the same final black hole (see figure 3 in that paper).

2.1.2. Frequency range and limitations: The numerical waveforms used in this paper contain a small number of cycles prior to merger; and therefore, they do not span the entire LIGO frequency band. The initial orbital period of these runs is chosen to be the same within numerical error. Due to the proximity to the merger, the definition of eccentricity is 
ambiguous. An additional effect is that the higher eccentricity runs merged faster than the lower eccentricities, and have fewer cycles prior to merger.

The stringent lower limit on $f_{\min }$, the lower end of the spectrum of the waveform and hence also the data analysis, is $0.02 / M$ for the high eccentricity $e=0.6$, while for eccentricities $\leqslant 0.3$ the chosen cutoff is at $0.01 / M$. The orbital frequency oscillates due to precession and the frequency at the peri-astron is what is chosen for picking the cutoff. The mass for which the entire signal is in the LIGO band thus changes for each eccentricity. This is illustrated by the figure on the right in figure 1 . One can see from the figure that the late merger and ringdown signal will be 'in-band' for masses $\leqslant 500 M_{\odot}$ and out of band for higher masses, for the initial LIGO noise curve. Our results are reliable for masses $\geqslant 60 M_{\odot}$ for lower eccentricities and for masses $\geqslant 120 M_{\odot}$ for higher eccentricities, assuming initial LIGO noise. However, the results for higher eccentricities can be valid for lower masses by setting $f_{\min }$ to be proportionately higher, at say $100 \mathrm{~Hz}$ instead of $40 \mathrm{~Hz}$ for example.

\subsection{Caveats owing to definition of eccentricity:}

As highlighted in $[8,13]$, in the evolutions we use, as the eccentricity is increased there is a transition from inspiral to plunge at around $e \sim 0.5$. Actually without specifying the initial separation along with the orbital period/eccentricity as done in [8], the eccentricity by itself would not be a meaningful parameter. These runs then comprise a set of points along a onedimensional curve in the two-dimensional space of eccentricity and initial separation. The specific values of eccentricity then act as mere labels to represent a set of possible evolutions rather than being representative of eccentric evolutions in general.

\section{Results: distance reach of eccentric binary black holes}

We study the relative contribution to the signal-to-noise to the spin weighted spherical harmonic components of the eccentric waveforms in what follows.

\subsection{Signal to noise ratio (SNR)}

In the usual matched filtering analysis for gravitational wave detection, given two waveforms $\tilde{h}_{1}(f)$ and $\tilde{h}_{2}(f)$, the scalar (or inner) product between these two functions is defined as

$$
\left\langle h_{1} \mid h_{2}\right\rangle=4 \operatorname{Re} \int_{f_{\min }}^{f_{\max }} \frac{\tilde{h}_{1}(f) \tilde{h}_{2}^{*}(f)}{S_{h}(f)} \mathrm{d} f,
$$

where $S_{h}(f)$ denotes the noise spectrum for which we use the initial LIGO (iLIGO) noise curves.

The signal-to-noise ratio is given by

$$
\rho^{2}=\langle h \mid h\rangle
$$

and depends on the distance of the source from the detector.

\subsection{The role of higher spherical harmonic modes}

An estimate of the relative strength of signal-to-noise in the various spherical harmonic modes can give insight on whether or not they impact the detection. In general, owing to the asymmetry relative to the circular case, one would expect more signal in the higher modes of an eccentric binary. A general signal will combine various spherical harmonic modes depending on the inclination of the source relative to the observer. 

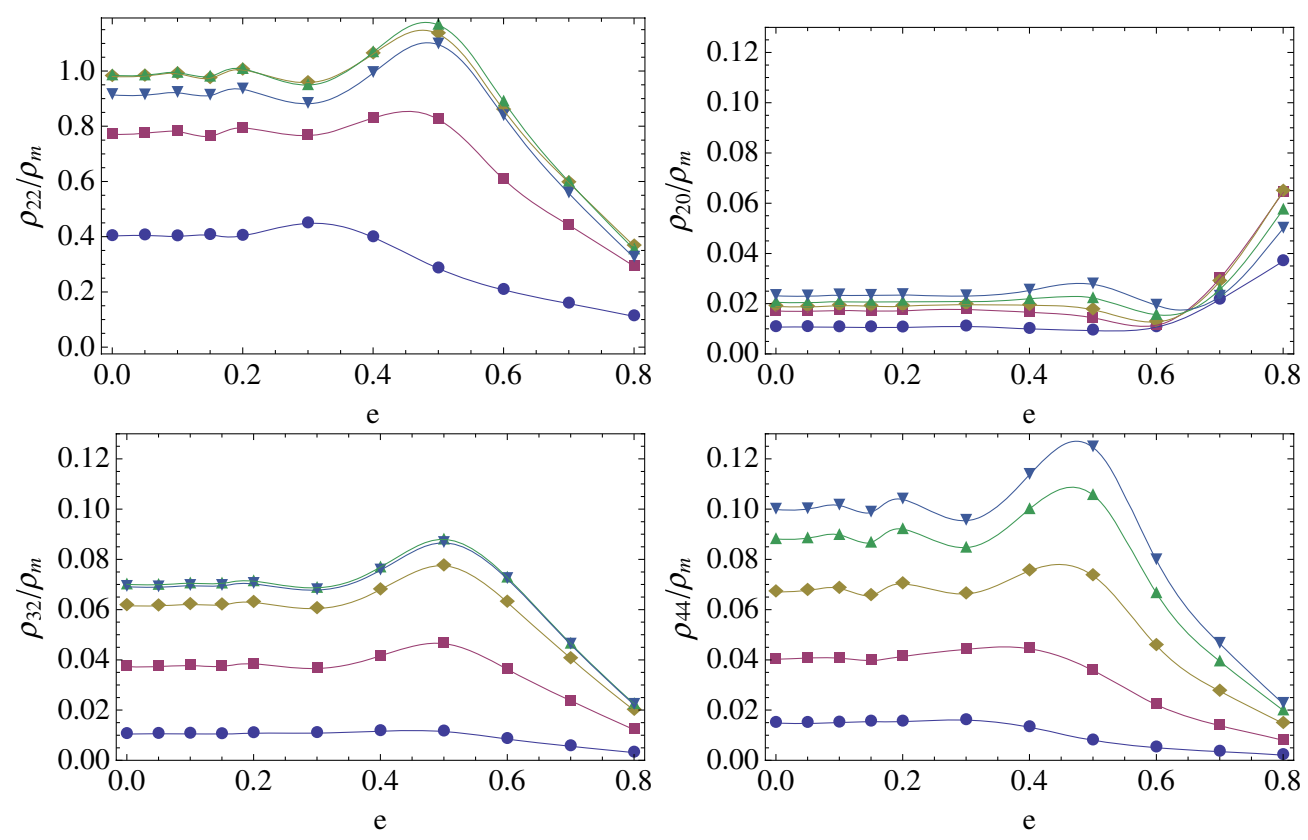

Figure 2. The relative signal-to-noise ratio for iLIGO in different modes of the eccentric waveforms. The dots, squares, vertical triangles, diamonds and inverted triangles represent the total masses $40,80,120,160$ and $200 M_{\odot}$ respectively. The top left/right figure is for $(\ell, m)=(2,2) /(2,0)$ and the bottom left/right is for $(3,2) /(4,4)$ modes respectively.

Figure 2 is a plot of the signal-to-noise ratio as a function of initial eccentricity for a selection of total mass values. The signal strength is inversely proportional to the distance of the source from the detector. We want to compare the mode variation with eccentricity and mass alone, so we scale out the distance. To remove the distance dependence, we normalize the SNR by the peak SNR of the $e=0$ waveform. This peak occurs at $\sim 140 M_{\odot}$. By normalizing our SNR in this manner we are comparing all the eccentric binaries at the same (arbitrary) distance. In other words, we compare sources with different eccentricities, a given mass and an arbitrary distance. The modes not shown here have strengths below the numerical error.

Figure 2 shows several facets of the SNR distribution into higher harmonics. The SNR in the $\ell=|m|=4$ mode has a significantly different variation with mass than the dominant, $\ell=|m|=2$ mode. This is because its $f_{\min }$ and $f_{\max }$ frequencies are twice that of the dominant mode, hence falling in a different place in the LIGO noise curve. The transition between inspiral and plunge occurs at $e \approx 0.5$ and the SNR around that is consistently higher than the circular case by $\sim 20 \%$. The $\ell=2, m=0$ mode contributes three times more in the nearly plunging case of $e=0.8$, while for $e \leqslant 0.3$, its contribution is more or less independent of eccentricities. The $\ell=3,|m|=2$ and $\ell=|m|=4$ modes exhibit a greater variation with mass than the $\ell=|m|=2$ mode.

Figure 2 also shows that the $\ell=|m|=4, \ell=3,|m|=2$ and $\ell=2, m=0$ are secondary. Because these modes carry less than $\approx 10 \%$ of the signal in general, we only consider the dominant mode in our further analysis. By disregarding the contribution of the secondary modes, we are incurring an error of approximately ten percent, the error being 


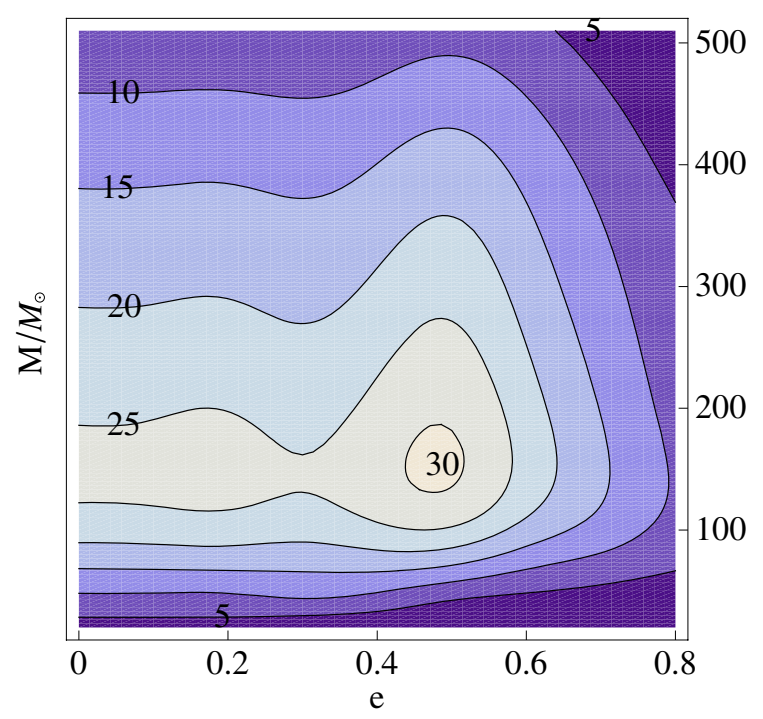

Figure 3. For a threshold iLIGO signal-to-noise of 10, the interpolated contours of the maximum distance reach (also called the signal horizon) in the (mass, eccentricity) parameter space. The contours are labeled in units of $10 \mathrm{Mpc}$ to facilitate display.

maximum near the inspiral-plunge transition eccentricity. In addition, there is interference between the modes that can add constructively or destructively to the SNR.

In order to estimate the extent of such interference, we calculated the fitting factors between the full waveform at various inclination angles which contained modes up to $\ell=4$ and a waveform with just the $\ell=|m|=2$ mode and found that they were within 0.95 for $e \leqslant 0.2$ at $100 M_{\odot}$. This indicates that at least for low eccentricities, the signal to noise ratios and hence the distance reach calculated using the dominant mode would be accurate to $5 \%$ even for an arbitrarily inclined source.

Ignoring the contribution of higher spherical harmonic modes is, within numerical error, the same as assuming an optimally oriented source. This is because the odd $\ell$ modes do not contribute in the case of such a source and the $\ell=4,|m|=2$ mode which will be the next dominant contribution is below numerical error for our case. The contribution of the $(4,4)$ mode is suppressed because of the spherical harmonic coefficients which are nonzero only for $m=2$. We ignore the $(3,2)$ mode as the error incurred by that is below the $5 \%$ level.

Hence, the assumption that the dominant $\ell=|m|=2$ mode is the same as the optimally oriented signal is a valid approximation. We study the properties of the dominant modes for various eccentricities and masses in the next section.

\subsection{Distance reach for optimally oriented eccentric binaries}

In figure 3 we remove the normalization to the peak of the circular SNR and plot contours of SNR with the total mass of the binary and eccentricity forming the axes. The SNR is inversely proportional to distance. Hence, this figure can be interpreted as the SNR for sources with different eccentricities and masses at a fixed distance of $100 \mathrm{Mpc}$, or equivalently, the contours of the distance at which the SNR would be 10 for each source. In the second case, the contours labels are to be read in units of $10 \mathrm{Mpc}$. 


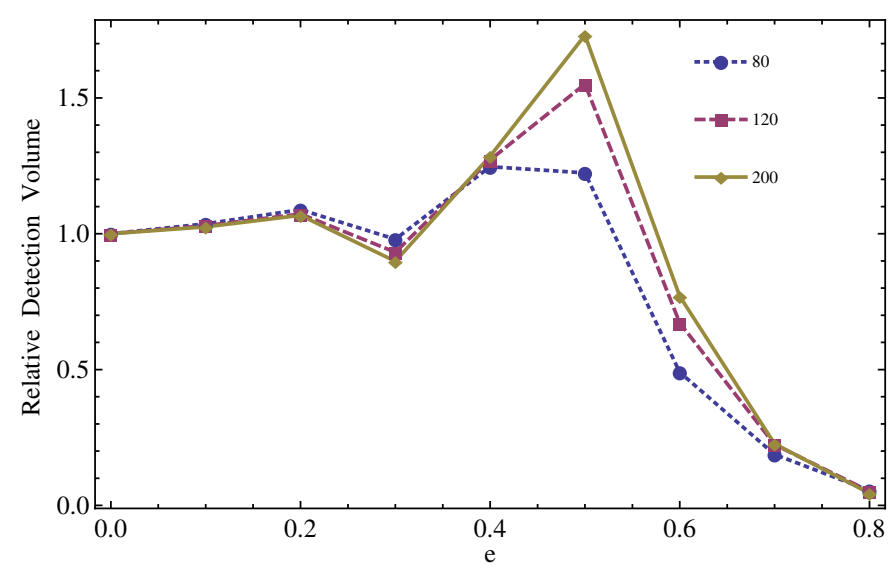

Figure 4. The relative enhancement in the detection volume due to eccentricity for three different masses, 80, 120, $200 M_{\odot}$ for iLIGO.

There is significantly more signal from the transition eccentricity than a quasi-circular binary at identical distance. This relationship can be inverted to infer that eccentric binaries of $e \sim 0.5$ can be seen to slightly greater distance, about $20-25 \%$ farther, than a circular binary. However, again note that the numerical evolutions initiated with this eccentricity and lead to plunging orbits, and the characterization of eccentricity is ill-defined for these cases. This enhancement suggests that at transition eccentricities which are not low enough for inspiral nor high enough for plunges, greater energy may be radiated and hence higher signal-to-noise ratios maybe observed.

This eccentricity range is interesting for two related reasons, it is the transition point to plunging orbits and the point where we found the maximum value of spin in the final black hole. However, our eccentric waveforms are not all equally accurate due to the specific initial data scheme used to set the initial eccentricity. At higher eccentricities, each orbit contributes energy at various frequencies and so having a relatively close-separated evolution may limit the accuracy of the signal. The effect may be acute at high eccentricities, where effectively one is capturing the last cycle of a long evolution, which is challenging to evolve in practice given the huge run time required.

\subsection{Relative enhancement of detection volume}

Given a threshold SNR of say ten, the maximum distance to which one can see a source determines a detection volume, assuming isotropy of the SNR with respect to source sky location. In order to show how dramatic the enhancement of the SNR shown in figure 3 is, we plot below the relative enhancement in the detection volume compared to that of the $e=0$ signals. This is given by $V_{\text {rel }}=(\rho(e, M) / \rho(0, M))^{3}$.

As we see from the figure 4 , the transition from inspiral to plunge leads to enhancement of the detection volume by almost a factor of two. As a result if one were to estimate a detection rate for these objects assuming a flat prior in eccentricity, the rate would be maximum for terminal eccentricities that range around $e \approx 0.5$. How robust this result is to change in initial data remains to be seen. 


\section{Conclusion}

The question of how eccentricity in the last few orbits of an intermediate mass black hole binary affects its distance range and signal-to-noise distribution in various higher modes is explored in this preliminary work. We examined this question using numerical relativity waveforms from eccentric binaries ranging from quasi-circular up to high eccentricities ranging from 0 to 0.8 in steps of 0.1 . The eccentricity is not well defined at $e \geqslant 0.5$ that leads to plunges rather than an orbit before merger, and those higher eccentricity values are to be considered only as a label for the runs. A more thorough treatment would require looking at longer evolutions of these high eccentricities.

A preliminary investigation into the importance that the higher spherical harmonic modes might have in signal from these eccentric binaries, found that the modes sub-dominant to $\ell=|m|=2$ carry less than $10 \%$ signal. The $\ell=|m|=4$ and the $\ell=3,|m|=2$ modes showed an enhancement in the signal-to-noise at the transition eccentricity $(e \sim 0.5)$. These modes do not contribute significantly to an optimally oriented binary.

Next we studied the signal-to-noise ratio variation assuming optimal source orientation and found that it peaks around the inspiral-plunge transition eccentricity $e \approx 0.5$. This results in being able to see an eccentric binary with eccentricities in the regime $(0.4,0.55)$ farther out than one can see an $e=0$ binary. However, the specific values of eccentricities here are not as important as the indication that the initial eccentricities which are at the transition seem to lead to a higher signal to noise ratio. The reduction in SNR in the case of plunges seems to be well known for the case of EMRI signals for example [14] but we explicitly show this to be the case even in the fully non-perturbative numerical relativity regime. More investigation of transition eccentricities would be needed before this conclusion can be made robust.

These values of SNR and distance reached were calculated assuming matched filtering of signals from eccentric sources with templates identical to the signal. Relaxing this assumption, one can ask how efficient are circular templates in capturing signals from eccentric sources. For low eccentricity stellar mass signals, such templates seem to be good enough for detection. However, for the case of IMBBH mergers, this question has not been settled yet.

In an upcoming paper [11], we shall study the efficiency of circular templates in capturing eccentric mergers of intermediate mass black hole binaries of mass $\geqslant 50 M_{\odot}$. We will also examine the effect of numerical errors in the eccentric numerical relativity runs and the effect they have on the distance reach etc. Another important avenue of investigation is to fill in the gap between stellar mass eccentric binary waveforms and the intermediate mass ones, which will likely require reliable hybrid waveforms constructed from numerical relativity and postNewtonian methods. Having such waveforms will allow us to make a definitive claim about the efficiency of circular templates. Finally, these analyses and conclusions were done using equal-mass, non-spinning waveforms. How unequal masses and spins break the degeneracy remains an interesting question for future work.

\section{Acknowledgments}

BV thanks Soumya Mohanty and Matt Benacquista for stimulating discussions, Patrick Brady for his insightful questions, Mario Diaz for encouragement and the Center for Gravitational Wave Astronomy, University of Texas at Brownsville for travel support and research funding. The Center for Gravitational Wave Physics at Penn State, where this project was started, is supported by the NSF under cooperative agreement PHY-0114375. Support for this work was also provided by NSF grants PHY-0653443 and PHY-0653303. Computations were performed 
at NCSA and TACC under allocation TG-PHY060013N. We thank an anonymous referee for carefully reading the paper and their detailed suggestions for improving the presentation.

\section{References}

[1] Amaro-Seoane P and Freitag M 2006 Intermediate-mass black holes in colliding clusters: implications for lower-frequency gravitational-wave astronomy Astrophys. J. 653 L53-6

[2] Fregeau J M, Larson S L, Miller M C, O’Shaughnessy R W and Rasio F A 2006 Observing IMBH IMBH binary coalescences via gravitational radiation Astrophys. J. $646 \mathrm{~L} 135-8$

[3] Amaro-Seoane P, Miller C and Freitag M 2009 Gravitational waves from eccentric intermediate-mass black hole binaries Astrophys. J. 692 L50-3

[4] O’Leary R M, Kocsis B and Loeb A 2009 Mon. Not. R. Astron. Soc. 395 2127-2146

[5] Martel K and Poisson E 1999 Gravitational waves from eccentric compact binaries: reduction in signal-to-noise ratio due to nonoptimal signal processing Phys. Rev. D 60124008

[6] Tessmer M and Gopakumar A 2007 Gravitational waves from compact binaries inspiralling along post-newtonian accurate eccentric orbits: data analysis implications

[7] Cokelaer T and Pathak D 2009 Searching for gravitational-wave signals emitted by eccentric compact binaries using a non-eccentric template bank: implications for ground-based detectors Class. Quantum Grav. $26045013-+$

[8] Hinder I, Vaishnav B, Herrmann F, Shoemaker D and Laguna P 2008 Circularization and final spin in eccentric binary black hole inspirals Phys. Rev. D 77081502

[9] Husa S, Hinder I and Lechner C 2006 Kranc: a mathematica application to generate numerical codes for tensorial evolution equations Comput. Phys. Commun. 174 983-1004

[10] Vaishnav B D 2008 Gravitational waves from intermediate mass binary black holes: first steps toward using numerical relativity waveforms for LIGO data analysis PhD Thesis Pennsylvania State University

[11] Vaishnav B, Hinder I, Shoemaker D and Herrmann F Observing eccentric intermediate mass binary black hole mergers with ligo (in preparation)

[12] Berti E, Cardoso V, Gonzalez J A, Sperhake U, Hannam M, Husa S and Bruegmann B 2007 Inspiral, merger and ringdown of unequal mass black hole binaries: a multipolar analysis Phys. Rev. D 76064034

[13] Sperhake U, Berti E, Cardoso V, Gonzalez J A, Brugmann B and Ansorg M 2008 Eccentric binary black-hole mergers: the transition from inspiral to plunge in general relativity Phys. Rev. D 78064069

[14] Barack L and Cutler C 2004 Confusion noise from lisa capture sources Phys. Rev. D 70122002 